\title{
单分散䍩基化 $\mathrm{Fe}_{3} \mathrm{O}_{4}$ 磁性纳米粒子的制备及表征
}

\author{
靳艳艳 ${ }^{(1)}$, 程武 ${ }^{(1)}$ ，王苗 ${ }^{(1)}$, 邢艳 ${ }^{\mathbb{1}}$ ，彭明丽 ${ }^{(12)^{*}}$ ，王小芳 ${ }^{(1)}$ 陈超 ${ }^{(2)}$ ，崔亚丽 ${ }^{(2 *}$ \\ (1) 西北大学化学与材料科学学院, 合成与天然功能分子化学教育部重点实验室, 西安 710069; \\ (2) 国家微检测系统工程技术研究中心, 西安 710069 \\ *联系人, E-mail: mlpeng@nwu.edu.cn; yalicui@nwu.edu.cn \\ 2013-06-03 收稿, 2013-09-16 接受, 2014-05-23 网络版发表 \\ 国家自然科学基金(21303136)和陕西省自然科学基金基础研究计划(2012JQ4026)资助
}

\begin{abstract}
摘要 单分散羧基功能化 $\mathrm{Fe}_{3} \mathrm{O}_{4}$ 磁性纳米粒子在生物医学诊断、治疗的基础和应用研究中具有 重要的意义. 本文在制备油酸稳定的磁性纳米粒子基础上, 利用高碘酸钠将其表面油酸充分氧 化制备了单分散羒基化 $\mathrm{Fe}_{3} \mathrm{O}_{4}$ 磁性纳米粒子. 采用傅里叶红外光谱仪(FT-IR)、透射电子显微镜 (TEM)、热失重分析(TGA)、X 射线粉末衍射仪(XRD)、振动样品磁强计(VSM)等方法对 $\mathrm{Fe}_{3} \mathrm{O}_{4}$ 磁性纳米粒子的形貌、大小、组成、磁强度以及分散性进行了表征. 结果表明, 该磁性纳米粒 子在常温下有良好的超顺磁性, 表面含有羒基, 直径为 $12 \mathrm{~nm}$, 粒径均一, 在水中分散良好. 利用 热重法和 4-溴甲基-6,7-二甲氧基香豆素化学反应方法测定其羒基含量均在 $10^{-7} \mathrm{~mol} / \mathrm{mg}$ 数量级.
\end{abstract}

\section{关键词}

单分散

磁性纳米粒子

氧化

羧基

含量测定
近年来, 具有单分散、高饱和磁化强度、超顺磁 性以及表面带有功能基团的磁性纳米粒子因其在后 续修饰及应用中显示出几乎相同的物理、化学和生物 学性质, 为其在生物医学诊断、治疗方面的基础和应 用研究提供了新的机遇 ${ }^{[1 \sim 3]}$. 磁性纳米粒子表面的功 能基团如羧基、胺基和羟基等, 不仅使磁性纳米粒子 在水溶液中具有良好的稳定性及分散性, 而且可以 用于偶联生物大分子 ${ }^{[4 \sim 7]}$, 如特异性靶向分子、药物

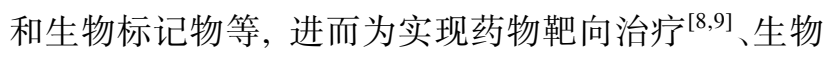

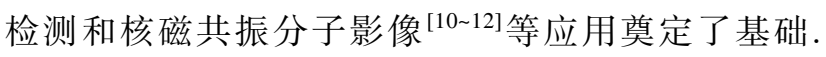
在众多表面修饰功能团中, 羧基是一种理想的能够 与生物大分子相互偶联的基团, 因此对高质量单分 散羧基功能化磁性氧化铁纳米粒子进行制备和性质 研究非常必要. $\mathrm{Hu}$ 等人 ${ }^{[13]}$ 采用原位合成法成功地制 备了表面带有羧基残基的生物相容性磁性氧化铁纳 米粒子. 苏鹏飞等人 ${ }^{[14]}$ 利用高锰酸钾氧化油酸法制 备了羧基化的磁性纳米粒子. 但这些方法得到的纳 米粒子的形貌和粒径均匀程度有待改善. 另外, 多个 课题组报道了利用小分子如谷胱甘肽 $(\mathrm{GSH})^{[15]}$ 、颈基
乙 酸 ${ }^{[16]}$ 和二巯基丁二酸 ${ }^{[17]}$ 等配体和磁性粒子表面的 配体进行交换, 或利用高聚物如聚乳酸(PLA $)^{[18]}$ 、聚 丙烯酸 $(\mathrm{PAA})^{[19,20]}$ 和马来酸䣶-1-十八碳烯交替共聚 物 $(\mathrm{PMAO})^{[21,22]}$ 等对磁性纳米粒子表面进行包覆修 饰, 制备了水溶性单分散羧基功能化磁性氧化铁纳 米粒子. 但这两种制备方法存在交换效率低且程度 难以控制、水合粒径明显增加、磁响应性减弱以及分 散性差等问题, 为其在生物医学领域的应用带来不 便. 本文报道了一种首先制备油酸稳定的磁性纳米 粒子, 然后利用高碘酸钠氧化磁性纳米粒子表面油 酸来制备羧基功能化单分散 $\mathrm{Fe}_{3} \mathrm{O}_{4}$ 磁性纳米颗粒的方

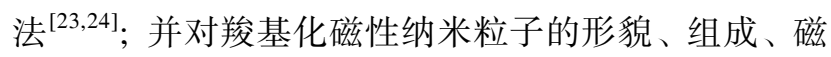
强度和表面羧基含量等进行了分析表征.

\section{1 实验}

(i ) 试剂与仪器. 无水乙醇、正己烷(AR, 天津 科密欧公司, 中国); 油酸(AR, 天津福晨化学试剂厂, 中国)；1-十八烯烃、乙酸乙酯、 $N, N$-二甲基甲酰胺 (DMF)、4-溴甲基-6,7-二甲氧基香豆素 (BMMC, AR,

引用格式: 靳艳艳, 程武, 王苗, 等. 单分散羧基化 $\mathrm{Fe}_{3} \mathrm{O}_{4}$ 磁性纳米粒子制备及表征. 科学通报, 2014, 59: 1700-1706

Jin Y Y, Cheng W, Wang M, et al. Synthesis and characterization of carboxy-functionalized monodisperse $\mathrm{Fe}_{3} \mathrm{O}_{4}$ magnetic nanoparticles (in Chinese). Chin Sci Bull (Chin Ver), 2014, 59: 1700-1706, doi: 10.1360/972013-663 
Sigma-Aldrich, 美国); 乙醚(AR, 天津富宇公司, 中 国); 乙腈(AR, Burdick \& Jackson, 美国); 高碘酸钠 (AR, 国药集团, 中国); 超纯水 $(\kappa=18.24 \mathrm{M} \Omega \mathrm{cm}$, 实 验室自制).

分析天平(AL204, Metter Toledo, 瑞士)、傅里叶红外光谱分析仪(FT-IR, Nicolet 5700, Thermo Electron, 美国)、透射电子显微镜(TEM, H-600, Hitachi, 日本)、 紫外-可见分光光度计(UV-Vis, UV-2550, Shimadzu, 日本)、热分析仪(TGA, 851e, Mettler, 瑞士)、X 射线 粉末衍射仪 (XRD, D/MAX-3C, Rigaku, 日本)、振动 样品磁强计(VSM, HH-15, 南京南大仪器厂, 中国).

(ii) 羧基功能化 $\mathrm{Fe}_{3} \mathrm{O}_{4}$ 磁性纳米粒子的合成. 将油酸铁复合物 $6.0 \mathrm{~g}^{[25]}, 1$-十八烯烃 $45 \mathrm{~mL}$ 及油酸 $1.5 \mathrm{~mL}$ 搅拌混匀, 快速升温到 $280 \sim 290^{\circ} \mathrm{C}$ 后, 调解升 温速度缓慢升至 $320^{\circ} \mathrm{C}$, 反应 $40 \mathrm{~min}$ 得到黑色混合物. 用乙醚和乙醇洗涤多次后分散于正已烷中. 取 $10 \mathrm{~mL}$ 含 $100 \mathrm{mg}$ 磁性纳米粒子的上述正已烷溶液与 $20 \mathrm{~mL}$ 体积比为 $1: 1$ 的乙酸乙酯/乙腈混合溶剂混合, 摚拌条 件下滴加 $\mathrm{NaIO}_{4}(0.41 \mathrm{~g}, 15 \mathrm{~mL})$ 水溶液, 室温反应 $2 \mathrm{~h}$. 磁性分离弃去上清溶液, 所得产物依次用乙醇、超纯 水洗涤多次, 后重新分散于超纯水中.

羧基含量的测定: BMMC 标准曲线的绘制. 准确 称取适量的 BMMC, 用体积比为 $1: 1 \mathrm{DMF}$ 水溶液进 行溶解, 转移至容量瓶定容, 配成浓度为 $1.00 \times 10^{-4}$ $\mathrm{mol} / \mathrm{L}$ 的溶液. 经稀释后配制出浓度为 $1.67 \times 10^{-5} \sim$ $1.00 \times 10^{-4} \mathrm{~mol} / \mathrm{L}$ 的 $0.005,0.010,0.015,0.020$ 和 0.030 $\mathrm{mg} / \mathrm{L}$ 标准工作液, 并测定各浓度溶液在 $350 \mathrm{~nm}$ 处的 吸光度, 绘制浓度-吸光度标准曲线. 羧基测定. 移 取 $0.1 \mathrm{mg}$ 磁性纳米粒子水溶液至 $2 \mathrm{~mL}$ 离心管中, 加 人含有 $1.6 \times 10^{-6} \mathrm{~mol}$ BMMC 的 DMF 水溶液 $1.8 \mathrm{~mL}$ 后, 超声混匀, 置于 $70^{\circ} \mathrm{C}$ 水中反应 $2 \mathrm{~h}$, 反应结束后 进行磁性分离, 再用 $1.8 \mathrm{~mL}$ 的 DMF 水溶液分 3 次洗 涤, 合并上清溶液, 混匀后取 $0.3 \mathrm{~mL}$ 稀释至 $4 \mathrm{~mL}$, 测 定其在 $350 \mathrm{~nm}$ 处的吸光度.

\section{2 结果与讨论}

油酸为不饱和脂肪酸, 其在 C9 和 C10 之间有碳 碳双键, 因此利用氧化剂选择性氧化纳米粒子表面 油酸中的碳碳双键会得到具有不同功能基团的纳米 粒子. 例如, 利用含有高碘酸钠的勒米厄-冯.鲁德洛 夫(Lemieux-von Rudloff)试剂在水/环已烷/叔丁醇混 合溶剂中可以将纳米粒子表面油酸氧化为壬二酸,
从而得到羒基功能化的纳米粒子 ${ }^{[23]}$; 另有研究表明, 在不同当量的高碘酸钠作用下, 油酸能够被选择性 氧化为醛基或羧基 ${ }^{[24,26]}$. 因此, 在混合溶剂中利用过 量的高碘酸钠将磁性纳米粒子表面的油酸中碳碳双 键氧化为羧基, 可以得到羧基功能化磁性纳米粒子.

\subsection{FI-IR 分析}

油酸铁复合物、油酸稳定的磁性纳米粒子以及 羧基功能化磁性纳米粒子的FI-IR红外光谱如图 1所 示. 从图1(a)可以看出, 油酸铁复合物在 3440,3010 , $2925,2850,1710,1591$ 和 $1465 \mathrm{~cm}^{-1}$ 等处有分别来源 于- $\mathrm{OH}, \mathrm{C}=\mathrm{C}-\mathrm{H},-\mathrm{CH}_{2},-\mathrm{COOH}$ 以及 $\mathrm{COO}-\mathrm{Fe}$ 等的特 征振动峰, 其位置和强度与文献 [27]报道一致. 由图 1(b) 可知, 油酸稳定的磁性纳米粒子在 3440, $3010,2920,2850,1635$ 和 $1556 \mathrm{~cm}^{-1}$ 等处有吸收峰, 这与之前油酸铁复合物的吸收基本吻合, 说明磁性 纳米粒子的表面仍然吸附有油酸分子; 而 $596 \mathrm{~cm}^{-1}$ 处出现的 $\mathrm{Fe}-\mathrm{O}-\mathrm{Fe}$ 强振动峰表明了磁性 $\mathrm{Fe}_{3} \mathrm{O}_{4}$ 粒子的 形成，这些数据也与文献[28]报道相吻合. 与图 1(a), (b)对比可以看出, 图1(c)中在 $3450 \mathrm{~cm}^{-1}$ 处依然有宽 吸收峰, 亚甲基在 2920 和 $2850 \mathrm{~cm}^{-1}$ 处的吸收强度明 显减弱, 即 $-\mathrm{CH}_{2}$ 的含量较少; 且在 $3010 \mathrm{~cm}^{-1}$ 处的 $\mathrm{C}=\mathrm{C}-\mathrm{H}$ 振动峰未出现, 说明油酸几乎被全部氧化; 而在 1556 和 $596 \mathrm{~cm}^{-1}$ 处振动峰仍然存在, 表明磁性 纳米粒子及其表面的 $\mathrm{COO}-\mathrm{Fe}$ 化学键仍存在; 在 $1710 \mathrm{~cm}^{-1}$ 处再次出现羰基振动吸收峰, 说明磁性纳 米粒子表面存在 $-\mathrm{COOH}$, 即磁性纳米粒子表面具有

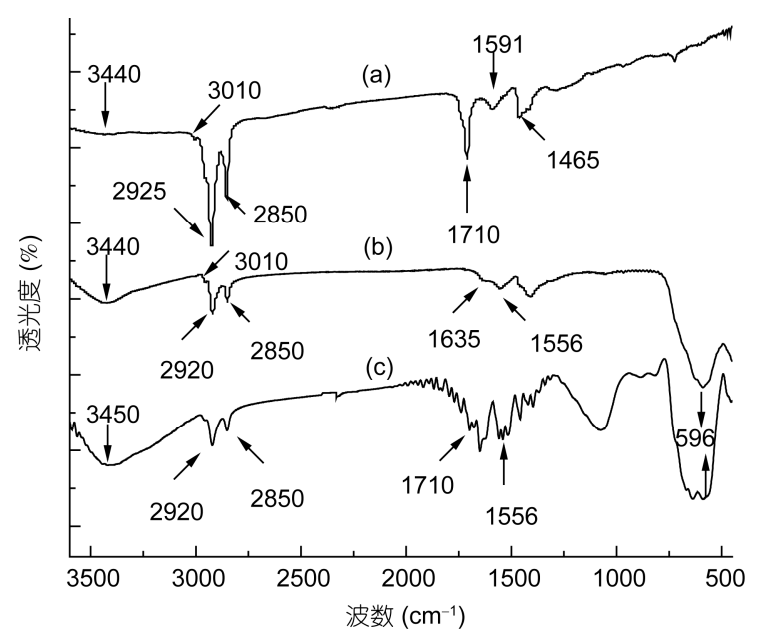

图 1 油酸铁复合物 $(\mathrm{a}) 、$ 油酸稳定 $\mathrm{Fe}_{3} \mathrm{O}_{4}$ 磁性纳米粒子(b) 和 羧基化 $\mathrm{Fe}_{3} \mathrm{O}_{4}$ 磁性纳米粒子(c)的 FT-IR 图谱 
羧基功能团.

\section{2 磁性纳米粒子的粒径表征}

利用 TEM 对油酸稳定的 $\mathrm{Fe}_{3} \mathrm{O}_{4}$ 磁性纳米粒子和 羧基化 $\mathrm{Fe}_{3} \mathrm{O}_{4}$ 磁性纳米粒子形貌、大小以及分散性进 行了研究, 结果如图 2 所示. 其中, 图 2(a)为油酸稳 定的 $\mathrm{Fe}_{3} \mathrm{O}_{4}$ 磁性纳米粒子分散在正己烷中的 TEM 图, 可以看出, 纳米粒子约为 $12 \mathrm{~nm}$, 粒径均匀, 单分散 分布, 在正已烷中分散良好; 图 2(b)为羒基化 $\mathrm{Fe}_{3} \mathrm{O}_{4}$ 磁性纳米粒子分散在水中的 TEM 图, 从图中可知, 该纳米粒子大小变化不明显, 粒径分布均匀. 该磁性 纳米颗粒水合粒径分布如图 2(c) 所示, 其平均粒径为 $24.1 \pm 2.1 \mathrm{~nm}$, 粒径分布为单峰, 说明羧基化 $\mathrm{Fe}_{3} \mathrm{O}_{4}$ 纳 米颗粒在水溶液中呈单分散分布且分散性较好. 利
用氧化方法制备的羧基化磁性纳米粒子保留了原来 油酸稳定磁性纳米粒子的单分散性.

\section{$2.3 \mathrm{XRD}$ 分析}

利用 $\mathrm{X}$ 射线粉末衍射仪对油酸稳定的 $\mathrm{Fe}_{3} \mathrm{O}_{4}$ 磁性 纳米粒子和羧基化 $\mathrm{Fe}_{3} \mathrm{O}_{4}$ 磁性纳米粒子粉末进行了表 征, 如图 3 所示. 由羧基化磁性纳米粒子 XRD 图谱(图 $3(\mathrm{a})$ ) 可知, 在衍射角分别为 $30.06^{\circ}, 35.50^{\circ}, 44.28^{\circ}$, $54.02^{\circ}, 57.12^{\circ}$ 和 $64.30^{\circ}$ 处均有明显的衍射特征峰, 衍 射角度和 $\mathrm{Fe}_{3} \mathrm{O}_{4}$ 标准图谱数据(JCPDS No.79-0418)相 吻合 ${ }^{[29]}$, 分别对应 $\mathrm{Fe}_{3} \mathrm{O}_{4}$ 晶体的(220), (311), (400), (422), (511)和(440)晶面. 图 3(a)和(b)中各个衍射峰对 应的衍射角大小相同, 说明在制备羒基化磁性纳米粒 子过程中使用氧化剂并未影响磁性纳米粒子的组分.
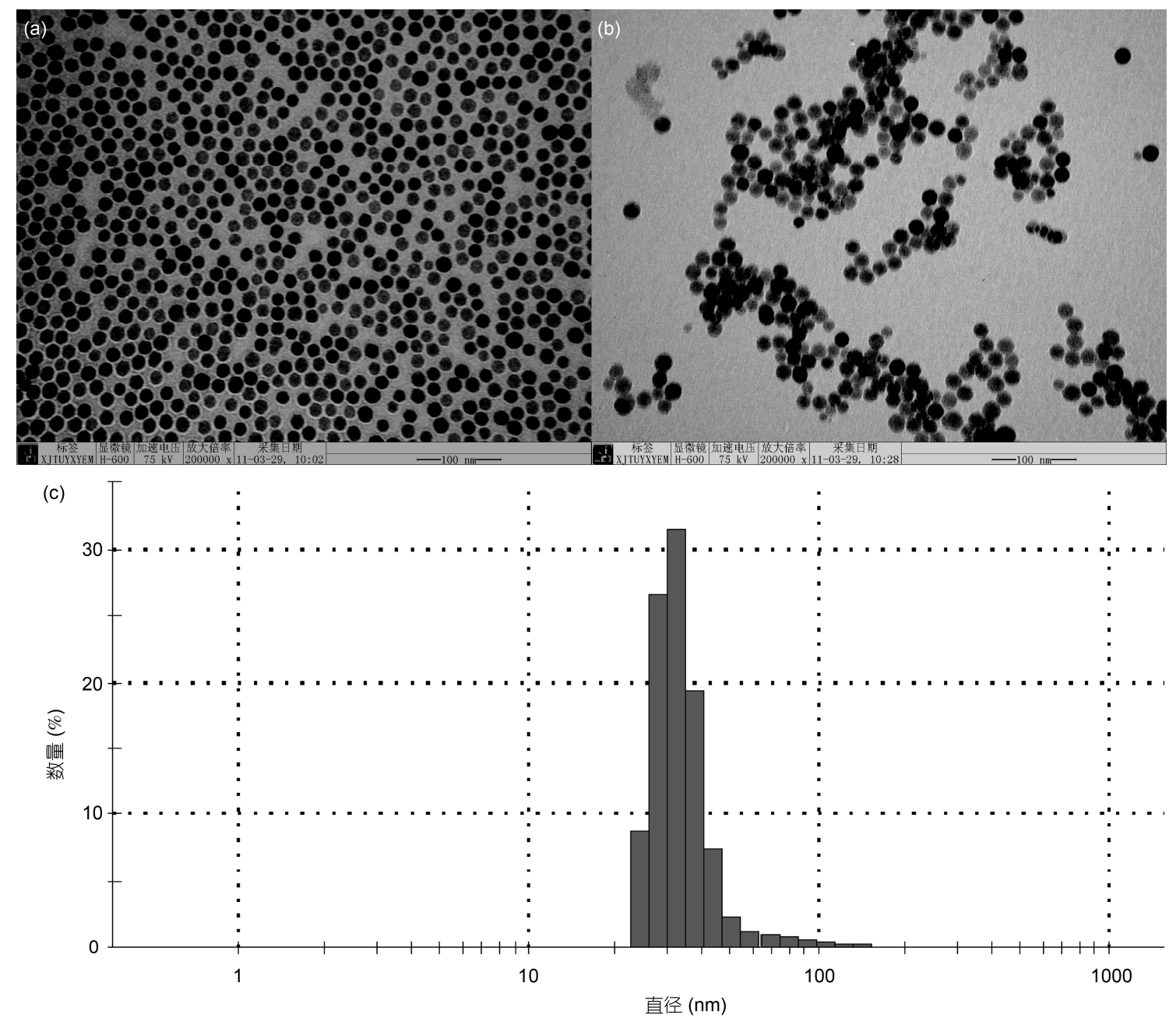

图 2 油酸稳定 $\mathrm{Fe}_{3} \mathrm{O}_{4}$ 磁性纳米粒子(a)、羧基化 $\mathrm{Fe}_{3} \mathrm{O}_{4}$ 磁性纳米粒子(b)的 $\mathrm{TEM}$ 图以及羧基化 $\mathrm{Fe}_{3} \mathrm{O}_{4}$ 纳米颗粒的 $\mathrm{DLS}$ 粒径 分布图(c) 


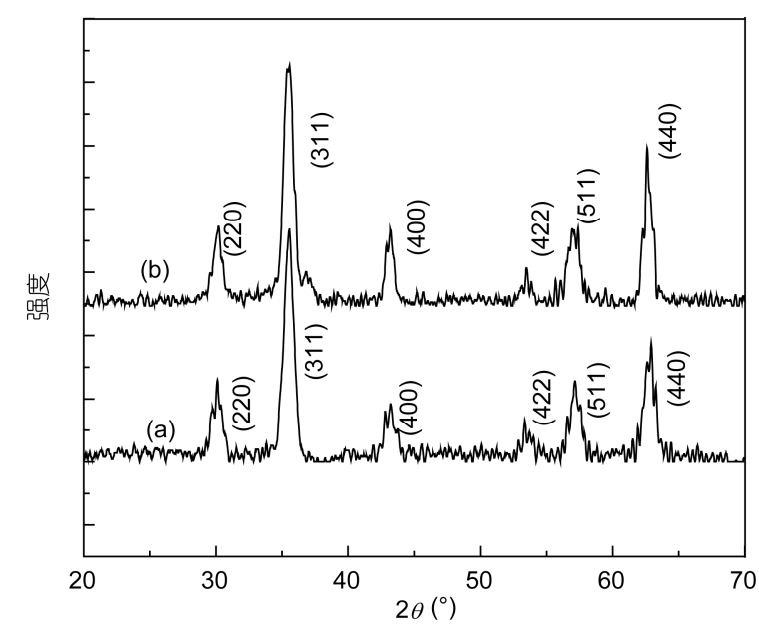

图 3 羧基化 $\mathrm{Fe}_{3} \mathrm{O}_{4}$ 磁性纳米粒子 $(\mathrm{a})$ 以及油酸稳定 $\mathrm{Fe}_{3} \mathrm{O}_{4}$ 磁性纳米粒子(b)的 XRD 图

\section{4 磁学性质表征}

采用 VSM 对油酸稳定的 $\mathrm{Fe}_{3} \mathrm{O}_{4}$ 磁性纳米粒子和羧 基化 $\mathrm{Fe}_{3} \mathrm{O}_{4}$ 磁性纳米粒子进行表征, 结果如图 4 所示. 从图中可以看出, 二者的饱和磁化强度曲线形状相似, 表现出良好的超顺磁性; 羧基化磁性纳米粒子与油酸 稳定的磁性纳米粒子的饱和磁化强度值分别为 47 和 $44.6 \mathrm{emu} / \mathrm{g}$, 说明实验中采用氧化来制备羧基化磁性纳 米粒子对材料的磁学性质影响不明显.

\section{5 表面羧基含量的测定}

纳米粒子表面功能基团的定量测定对后续磁性 纳米偶联生物大分子, 如抗体、蛋白质、核酸和多肽 等具有重要的指导意义. 目前, 磁性纳米粒子表面羧

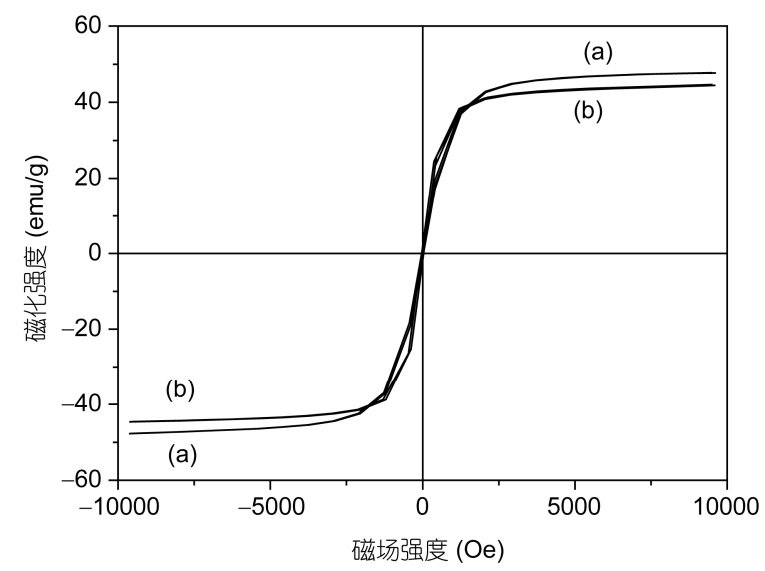

图 4 羧基化 $\mathrm{Fe}_{3} \mathrm{O}_{4}$ 磁性纳米粒子(a)以及油酸稳定 $\mathrm{Fe}_{3} \mathrm{O}_{4}$ 磁 性纳米粒子(b)的 VSM 图
基定量通常使用滴定法，如电导滴定法 ${ }^{[30]}$ 、酸碱滴定 法 $^{[31]}$, 或热分析技术如 TGA，等温滴定量热法(ITC) 等方法定量 ${ }^{[14]}$. 但这些方法往往滴定终点不明显、所 需样品量较大、受人为因素影响大, 不能满足对纳米 材料分析的需要. 因此, 本文利用 BMMC 与羧基能 够等化学计量反应的特点 ${ }^{[32]}$, 借助紫外-可见分光光 度计测定单分散磁性纳米颗粒表面的羧基含量, 并 将其与热失重分析法进行了对比.

（1）热失重测定羧基含量. 利用热失重仪对油 酸稳定的磁性纳米粒子和羧基化磁性纳米粒子进行 表征, 结果如图5所示. 由图5(a)可以看出, 油酸稳定 $\mathrm{Fe}_{3} \mathrm{O}_{4}$ 磁性纳米粒子在 $200 \sim 550^{\circ} \mathrm{C}$ 范围内失重明显, 失 重率为 $23.8 \%$, 这主要是由磁性粒子表面丢失吸附的 油酸造成. 而图 5(b) 显示, 羧基化磁性纳米粒子在 $100{ }^{\circ} \mathrm{C}$ 之前有 $4.3 \%$ 的少量失重, 该部分失重可能来源 于吸附水; 在 $200^{\circ} \mathrm{C}$ 短暂失重后, 快速到达平台区, 失 重率为 $15.7 \%$, 这比油酸稳定的磁性纳米粒子失重率 明显减少, 说明油酸发生分解生成了壬二酸. 另外, 根据式(1)计算可知, 磁性纳米粒子表面油酸和壬二酸 含量分别为 $8.43 \times 10^{-7}$ 和 $8.35 \times 10^{-7} \mathrm{~mol} / \mathrm{mg}$, 二者含量 基本相同, 说明油酸几乎全部发生氧化生成了壬二酸, 所以, 羧基化磁性纳米粒子表面羧基含量为 $8.35 \times 10^{-7}$ $\mathrm{mol} / \mathrm{mg}$.

$$
n=\frac{1.0 \times 10^{-3} \mathrm{am}}{M},
$$

式中, $a$ 为油酸稳定的磁性纳米粒子和羧基化磁性纳 米粒子的失重率值, $M$ 为壬二酸或油酸的相对分子质 量(油酸 $282.22 \mathrm{~g} / \mathrm{mol}$, 壬二酸 $188 \mathrm{~g} / \mathrm{mol}$ ), $m$ 为磁性纳 米粒子的质量 $(1 \mathrm{mg})$.

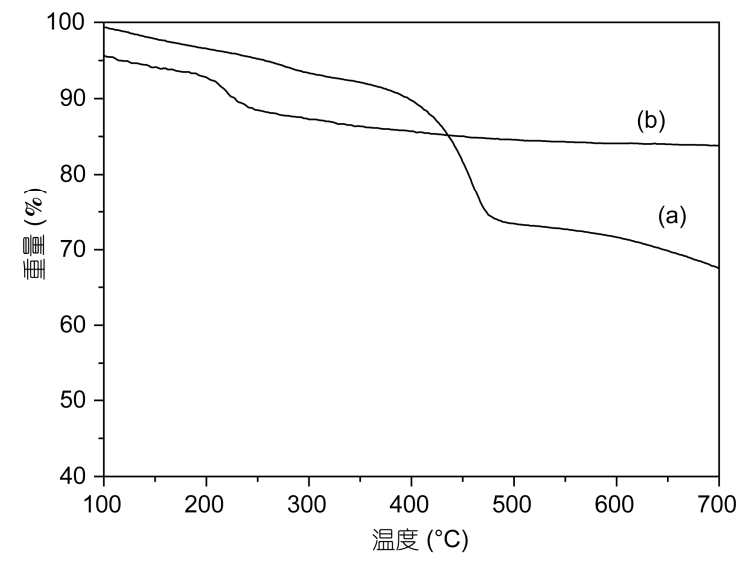

图 5 油酸稳定 $\mathrm{Fe}_{3} \mathrm{O}_{4}$ 磁性纳米粒子(a)以及羧基化 $\mathrm{Fe}_{3} \mathrm{O}_{4}$ 磁性纳米粒子(b)的 TGA 图 
(2) 偶联 BMMC 羧基含量的测定. (i ) 测定原 理. 在 DMF 和水的混合体系中, BMMC 的 4-烯丙基 溴能够与磁性纳米粒子表面羧基发生成酯反应. 根 据 BMMC 在反应前后含量的变化计算磁性纳米粒子 表面羧基含量, 其反应原理如图 6 所示 ${ }^{[32]}$. (ii) $\mathrm{BMMC}$ 标准曲线的建立. 图 7 所示为 BMMC 溶液的 紫外-可见吸收光谱图谱. BMMC 溶液在 $350 \mathrm{~nm}$ 处有 最大吸收峰, 因此, 在该波长下测定不同浓度 BMMC 标准工作液的吸光度, 并绘制成浓度一吸光度 标准曲线, 如图 7 插图所示. 从图中可以看出, 溶液 在浓度 $1.67 \times 10^{-5} \sim 1.00 \times 10^{-4} \mathrm{~mol} / \mathrm{L}$ 范围内符合郎伯比尔定律, 具有良好的线性关系. (iii) 羧基含量的测 定与计算. 用偶联 BMMC 测定羧基含量时进行 3 次 平行实验, 测定结果见表 1 . 可以看出, 该方法测得 的羧基化磁性纳米粒子表面羧基平均含量为 $11.7 \times 10^{-7} \mathrm{~mol} / \mathrm{mg}$, 比热重分析计算得到的羧基含量 $\left(8.35 \times 10^{-7} \mathrm{~mol} / \mathrm{mg}\right)$ 数值偏大, 这可能是因为即使经 过多次洗涤, BMMC 仍然会在磁性纳米粒子表面存 在少量的非特异性吸附. 但是该方法测定结果与热 重分析结果仍然良好地保持在一个数量级, 且测定 时所需磁性纳米粒子的数量较小 (仅 $0.1 \mathrm{mg}$ )、方法简 单, 因而能够更好地满足后续磁性纳米材料分析和 应用的要求。

\section{3 结论}

利用高碘酸钠将高温热解得到的油酸稳定的磁 性纳米粒子表面油酸进行氧化, 简便快捷地制备了 单分散羧基功能化的 $\mathrm{Fe}_{3} \mathrm{O}_{4}$ 磁性纳米粒子. 该磁性纳

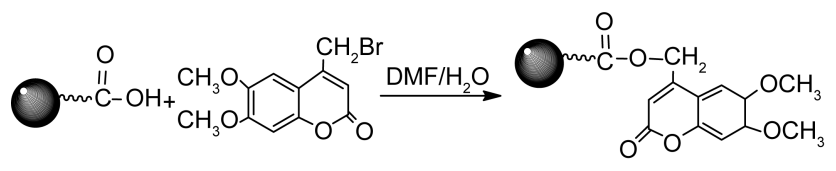

图 6 BMMC 与羧基化磁性纳米粒子表面羧基的反应原理图

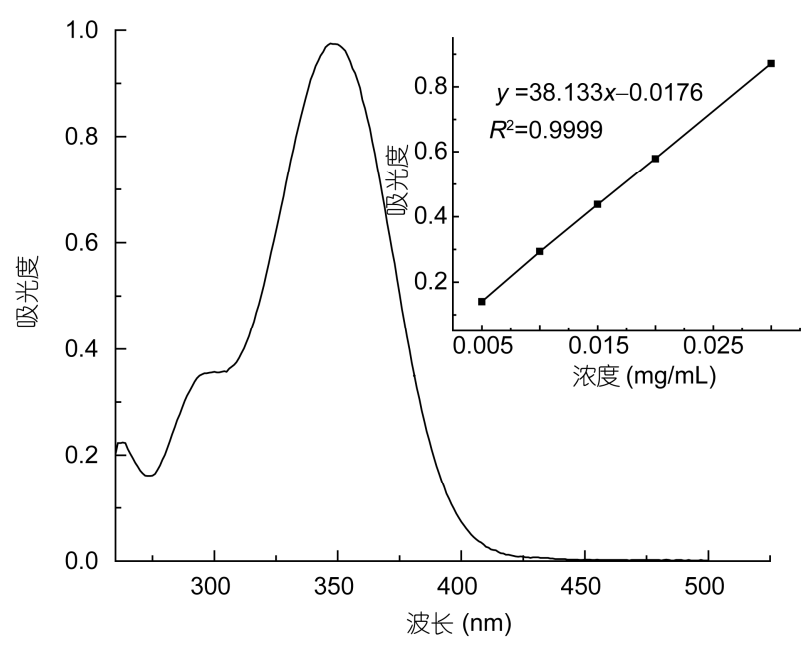

图 7 BMMC 的紫外-可见吸收光谱图 插图为标准曲线

表 1 平行实验测定羧基化磁性纳米粒子表面羧基含量

\begin{tabular}{ccc}
\hline 序号 & 吸光度 & 羧基含量 $\left(10^{-5} \mathrm{~mol}\right)$ \\
\hline 1 & 0.335 & 0.133 \\
2 & 0.331 & 0.117 \\
3 & 0.337 & 0.106 \\
平均值 & 0.334 & 0.117 \\
\hline
\end{tabular}

米粒子的 FI-IR 谱图显示, 其表面含有羧基官能团; TEM 结果显示, 该纳米粒子的粒径为 $12 \mathrm{~nm}$, 且粒径 均一, 为单分散分布、在水中分散良好; XRD 和 VSM 表征结果显示, 利用氧化方法制备羧基对磁性纳米 粒子的磁强度和成分等影响很小; 利用 TGA 和 BMMC 化学反应方法测定其羧基含量均为 $10^{-7} \mathrm{~mol} / \mathrm{mg}$ 数量级. 该工作不仅为制备单分散羧基化磁性氧化 铁纳米粒子及测定纳米材料表面羧基含量提供了新 的实验方法, 而且为靶向药物治疗、生物检测以及核 磁共振分子影像等应用中利用磁性颗粒偶联生物大 分子, 如特异性靶向分子、药物和生物标记物等的实 现奠定了基础.

\section{参考文献}

1 Jeong U, Teng X W, Wang Y. Superparamagnetic colloids: Controlled synthesis and niche applications. Adv Mater, 2007, 19: 33-60

2 黎汉生, 张东翔. 磁性催化剂研究进展. 材料导报, 2005, 8: 5-8

3 张吉林, 洪广言, 倪嘉缵。单分散磁性纳米粒子靶向药物载体. 化学进展, 2009, 21: 880-889

4 Parviz A, He X X, Wang K M, et al. An efficient method for recovery of target ssDNA based on amino-modified silica-coated magnetic nanoparticles. Talanta, 2005, 67: 548-554

5 间妍, 赵凌云, 王晓文, 等. 羧基磁流体制备和牛血清白蛋白表面修饰. 科技导报, 2010, 28: 73-76 
6 Wang X, Li Y D. Monodisperse nanocrystals: General synthesis, assembly, and their applications. Chem Commun, 2007, (2): 2901-2910

7 Tanaka T, Shibata K, Hosokawa M, et al. Characterization of magnetic nanoparticles modified with thiol functionalized PAMAM dendron for DNA recovery. J Colloid Interface Sci, 2012, 377: 469-475

8 Conroy S, Lee J S H, Zhang M Q. Magnetic nanoparticles in MR imaging and drug delivery. Adv Drug Delivery Rev, 2008, 60: 1252-1265

9 Chertok B, David A E, Yang V C. Polyethyleneimine-modified iron oxide nanoparticles for brain tumor drug delivery using magnetic targeting and intra-carotid administration. Biomaterials, 2010, 31: 6317-6324

10 Gu J H, Zhang W, Yang X L. Preparation of a superparamagnetic MRI contrast agent with a tumor targeting function. Mater Lett, 2013, 94: 8-10

11 Ferguson R M, Minard K R, Khandhar A P, et al. Optimizing magnetite nanoparticles for mass sensitivity in magnetic particle imaging. Med Phys, 2011, 38: 1619-1626

12 张霖倩, 吴秋芳, 陈雪梅, 等. 超顺磁性纳米氧化铁的制备、表面修饰及其在磁共振成像造影剂方面的应用. 材料导报, 2011, 25: $35-40$

13 Hu F Q, Li Z, Tu C F, et al. Preparation of magnetite nanocrystals with surfacereactive moieties by one-pot reaction. J Colloid Interface Sci, 2007, 311: 469-474

14 苏鹏飞, 陈国, 赵珺. 表面羧基化 $\mathrm{Fe}_{3} \mathrm{O}_{4}$ 磁性纳米粒子的快捷制备及表征. 高等学校化学学报, 2011,32: 1472-1477

15 鄢祝兵, 马勇杰, 古宏晨, 等. 谷胱甘肽纳米 $\mathrm{Fe}_{3} \mathrm{O}_{4}$ 对人肺腺癌细胞 SPC-A1 影响. 中国公共卫生, 2006, 22: 1445-1447

16 Woo K, Hong J. Surface modification of superparamagnetic iron oxide nanoparticles for clinical applications. IEEE T Magn, 2005, 14: 4137-4139

17 Huh Y M, Jun Y K, Song H T, et al. In vivo magnetic resonance detection of cancer by using multifunctional magnetic nanocrystals. J Am Chem Soc, 2005, 127: 12387-12391

18 Ren J, Hong H Y, Ren T B, et al. Preparation and characterization of magnetic PLA-PEG composite nanoparticles for drug targeting. React Funct Polym, 2006, 66: 944-951

19 Zhang T R, Ge J P, Hu Y X. A general approach for transferring hydrophobic nanocrystals into water. Nano Lett, 2007, 7: 3203-3207

20 Kim S W, Kim S, Tracy J B, et al. Phosphine oxide polymer for water-soluble nanoparticles. J Am Chem Soc, 2005, 127: 4556-4557

21 Yu W W, Chang E, Falkner J C. Forming biocompatible and nonaggregated nanocrystals in water using amphiphilic polymers. J Am Chem Soc, 2007, 129: 2871-2879

22 White M A, Johnson J A, Koberstein J T, et al. Toward the syntheses of universal ligands for metal oxide surfaces: Controlling surface functionality through click chemistry. J Am Chem Soc, 2006, 128: 11356-11357

23 Chen $\mathrm{Z} \mathrm{G}$, Chen $\mathrm{H} \mathrm{L}$, Hu H, et al. Versatile synthesis strategy for carboxylic acid functionalized upconverting nanophosphors as biological labels. J Am Chem Soc, 2008, 130: 3023-3029

24 Zimmermann F, Meux E, Mieloszynski J L, et al. Ruthenium catalysed oxidation without $\mathrm{CCl}_{4}$ of oleic acid, other monoenic fatty acids and alkenes. Tetrahedron Lett, 2005, 46: 3201-3203

25 Park J, An K, Hwang Y, et al. Ultra-large-scale syntheses of monodisperse nanocrystals. Nat Mater, 2004, 3: 891-895

26 Yang D, Zhang C. Ruthenium-catalyzed oxidative cleavage of olefins to aldehydes. J Org Chem, 2001, 66: 4814-4818

27 Zhang L, He R, Gu H C, et al. Oleic acid coating on the monodisperse magnetite nanoparticles. Appl Surf Sci, 2006, 253: 2611-2617

28 Palma R D, Peeters S, Van Bael M J, et al. Silane ligand exchange to make hydrophobic superparamagnetic nanoparticles waterdispersible. J Mater Chem, 2007, 19: 1821-1831

29 Zhang C Q, Cui Y L, Di D, et al. Solvothermal synthesis of uniform magnetite microspheres. Funct Mater Lett, 2010, 3: 125-129

30 贺枰, 崔陇兰, 强伟丽, 等. 高 $\mathrm{Fe}_{3} \mathrm{O}_{4}$ 含量单分散 $\mathrm{P}(\mathrm{St} / \mathrm{AA})$ 复合微球的合成与表征. 高分子学报, 2007, 8: 731-736

31 杨旭, 李欣, 潘复生, 等. 表面含羧基的磁性高分子微球的制备和表征. 化学世界, 2006, 5: 276-280

32 Markowitz M A, Schoen P E, Kust P, et al. Surface acidity and basicity of functionalized silica particles. Colloid Surf A-Physicochem Eng Asp, 1999, 150: 85-94 


\title{
Synthesis and characterization of carboxy-functionalized monodisperse $\mathrm{Fe}_{3} \mathrm{O}_{4}$ magnetic nanoparticles
}

\author{
JIN YanYan ${ }^{1}$, CHENG Wu ${ }^{1}$, WANG Miao ${ }^{1}$, XING Yan $^{1}$, PENG MingLi ${ }^{1,2}$, WANG XiaoFang ${ }^{1}$, \\ CHEN Chao ${ }^{2} \&$ CUI $\mathrm{YaLi}^{2}$ \\ ${ }^{1}$ Key Laboratory of Synthetic and Natural Functional Molecular Chemistry of Ministry of Education, College of Chemistry and Materials Science, \\ Northwest University, Xi'an 710069, China; \\ ${ }^{2}$ National Engineering Research Center for Miniaturized Detection Systems, Xi'an 710069, China
}

The functionalized monodisperse $\mathrm{Fe}_{3} \mathrm{O}_{4}$ magnetic nanoparticles are of importance in the field of biomedical detection and therapy. In this paper, the carboxyl-functionalized monodisperse $\mathrm{Fe}_{3} \mathrm{O}_{4}$ magnetic nanoparticles were synthesized by first synthesis of the oleic acid stabilized monodisperse iron oxide nanoparticles, and then the oxidation of oleic acid on the surface of the magnetic nanoparticles by sodium periodate. The nanoparticles were characterized by FT-IR, transmission electron microscopy (TEM), thermogravimetric analyzer (TGA), X-ray diffraction (XRD) and vibrating sample magnetometer (VSM). The experiment results show that the $\mathrm{Fe}_{3} \mathrm{O}_{4}$ magnetic nanoparticles have been functionalized by carboxyl group, and the diameter was $12 \mathrm{~nm}$ with superparamagnetism at room temperature momodisperse and good dispersion in aqueous solution. The concentration of carboxyl group on the surface of these nanoparticles was determined by TGA and 4-bromomethyl-6,7-dimethyloxycoumarin reaction method, respectively, the data show that the concentration of carboxyl group is on the order of $10^{-7} \mathrm{~mol} / \mathrm{mg}$.

monodisperse, magnetic nanoparticles, oxidation, carboxyl group, determination

doi: 10.1360/972013-663 